73rd Conference of the Italian Thermal Machines Engineering Association (ATI 2018), 12-14 September 2018, Pisa, Italy

\title{
Validation of a NOx estimation methodology based on the analysis of in-cylinder pressure trace
}

\author{
Filippo Carra ${ }^{\mathrm{a}}$, Fabrizio Pontia, Vittorio Ravagliolia* \\ ${ }^{a}$ University of Bologna, Forli, Italy
}

\begin{abstract}
Internal combustion engines control systems are evolving rapidly in order to meet the most recent emissions standards: this process requires a deep knowledge of how the combustion process takes place, since heat-release control is crucial to manage the trade-off between engine-out emissions and best suit the tailpipe aftertreatment system operating point.

Nitrogen oxides (NOx) have currently become a critical pollutant emission that needs to be limited in compressionignited engines. Since a selective catalytic reduction (SCR) system is present in several applications, engine-out NOx concentration is a fundamental parameter to be evaluated.

This work shows how an estimation of NOx concentration can be deduced from instantaneous in-cylinder pressure measurement and some of the parameters, related to the intake charge, currently available on electronic control units (ECU). A prediction model, based on Zeldovich mechanisms and Arrhenius rate of combustion is proposed, which exploits as main contributions: rate of heat release and adiabatic flame temperature. An experimental campaign (DOE) has been carried out on a diesel engine, varying the main engine control parameters, to tune the model in steady-state operating point. The predictive capability is then evaluated by feeding the model with a validation dataset in both steady state and transient condition. Finally, model response to measure uncertainties is discussed.
\end{abstract}

(C) 2018 The Authors. Published by Elsevier Ltd.

This is an open access article under the CC BY-NC-ND license (https://creativecommons.org/licenses/by-nc-nd/4.0/)

Selection and peer-review under responsibility of the scientific committee of the 73rd Conference of the Italian Thermal Machines Engineering Association (ATI 2018).

* Corresponding author.

E-mail address: vittorio.ravaglioli2@unibo.it 


\section{Introduction}

The management systems for modern Diesel engines are struggling to cope with the highly restrictive regulations dealing with emission limits: engine, aftertreatment layouts and control systems are gaining in both complexity and cost. The more limiting factor in conventional compression-ignited engine is the empirical relation that links nitrogen oxides (NOx) and particulate matter (PM) production, which means that engine calibration has always to be optimized to meet a trade-off between these two kinds of pollutant species. Several studies have been proposed dealing with combustion NOx estimation, which adopt different techniques [1,2]. In addition, engine-out NOx concentration is a key parameter in those applications which fit a selective catalytic reduction equipment $[3,4]$. In this context, a reliable cycle resolved estimation approach is an efficient method to overcome physical sensors response dynamic in a way that the aftertreatment devices can be optimally controlled. Simple black box models compatible with the performance of a commercial electronic control unit (ECU) are often used to express NOx concentration vs injection pattern and air management dependency, but such an approach need a great calibration effort and is hardly applicable when the considered factors lay outside the ranges reached during the model training phase, or some neglected interactions between these factors become relevant. On the contrary, a physic-based model can be a more effective methodology because, once the problem phenomenology is understood, the predictive capability of the model itself is enlarged, to some extent, to every operating condition. Drawbacks are commonly identified in computational cost and the relatively unconventional and expensive transducers that have to be installed, whose signal has to be acquired and processed by the engine ECU.

The aim of this work is to validate a model that allows managing engine-out NOx concentration, by linking their own production to the physical processes taking place during combustion. As it is well-known, according to Zel'dovich mechanism, thermal NO production is highly influenced by local in-chamber temperature and oxygen availability. These two information can be deduced by adding to the ordinary engine equipment an in-chamber pressure sensor. The starting point of the present discussion is the model developed by Arrègle et al. in [5] which is also the basis for some revised solutions proposed by the same research group [6,7] and others [8]. This model, as previously sketched, takes as main input in-cylinder pressure trace on angle basis: rate of heat release and adiabatic flame temperature are deduced from this data, in addition, mass air flow rate sensor (MAF) and oxygen sensor (UEGO) are used to determine charge oxygen fraction, which has a direct impact on the adiabatic flame temperature. An experimental campaign has been carried out on a conventional diesel engine to train the model and verify its behavior in steady-state conditions, then some modification concerning the starting model structure are proposed. Finally the model response in transient conditions and its sensitivity to sensors drift are evaluated.

\begin{tabular}{|lll|}
\hline \multicolumn{2}{|c|}{ Nomenclature } & \\
$\mathrm{b}_{1}$ & {$[\mathrm{~K} / \mathrm{deg}]$} & $\mathrm{k}_{3}$ sensitivity to CA50 \\
$\mathrm{b}_{2}$ & {$[\mathrm{~K}]$} & $\mathrm{k}_{3}$ offset with respect to CA50 \\
BMEP & {$[\mathrm{bar}]$} & Brake mean effective pressure \\
$\mathrm{CA} 50$ & {$[\mathrm{deg}]$} & Angle at which $50 \%$ of fuel is burned \\
$\mathrm{CHR}$ & {$[\mathrm{J}]$} & Cumulative heat release \\
DOE & & Design of experiment \\
ECU & & Electronic control unit \\
EGR & & Exhaust gas recirculation \\
EOC & {$[\mathrm{deg}]$} & End of combustion \\
HRR & {$[\mathrm{J} / \mathrm{deg}]$} & Heat release rate \\
$\mathrm{k}_{1}$ & {$[\mathrm{adm}]$} & Global scaling coefficient for NOx estimation \\
$\mathrm{k}_{2}$ & {$[\mathrm{adm}]$} & Tuneable exponent for engine speed contribution \\
\hline
\end{tabular}




\begin{tabular}{|lll|}
\hline $\mathrm{k}_{3}$ & {$[\mathrm{~K}]$} & Tuneable exponential coefficient for Arrhenius-like contribution \\
$\mathrm{LHV}$ & {$[\mathrm{J} / \mathrm{kg}]$} & Lower heating value \\
$\mathrm{LTC}$ & & Low temperature combustion \\
$\mathrm{m}_{\text {air }}$ & {$[\mathrm{mg} / \mathrm{str}]$} & Fresh air mass per stroke \\
$\mathrm{m}_{\text {diesel }}$ & {$[\mathrm{mg} / \mathrm{str}]$} & Injected fuel mass per stroke \\
$\mathrm{MAF}$ & {$[\mathrm{kg} / \mathrm{s}]$} & Air mass flow \\
$\mathrm{n}$ & {$[\mathrm{rpm}]$} & Engine speed \\
$\mathrm{NHRR}$ & {$[\mathrm{J} / \mathrm{deg}]$} & Net heat release rate \\
$\mathrm{NOx}$ & & Nitrogen Oxides \\
$\mathrm{NOx}$ & {$[\mathrm{mg}]$} & Exhaust NOx mass \\
$\mathrm{p}$ & {$[\mathrm{Pa}]$} & In-cylinder pressure \\
$\mathrm{p}_{\text {boost }}$ & {$[\mathrm{Pa}]$} & Boost pressure \\
$\mathrm{PM}$ & & Particulate matter \\
$\mathrm{ppm}$ & {$[\mathrm{adm}]$} & Parts per million mass \\
$\mathrm{RMSE}$ & & Root mean square error \\
$\mathrm{SCR}$ & & Selective catalytic reduction \\
$\mathrm{SOC}$ & {$[\mathrm{deg}]$} & Start of combustion \\
$\mathrm{SOI}$ & {$[\mathrm{deg}]$} & Start of injection \\
$\mathrm{T}_{\mathrm{ad}}$ & {$[\mathrm{K}]$} & Adiabatic flame temperature \\
$\mathrm{T}_{\text {boost }}$ & {$[\mathrm{K}]$} & Intake manifold temperature \\
$\mathrm{T}_{\text {mean }}$ & {$[\mathrm{K}]$} & Mean in-chamber temperature \\
$\mathrm{UEGO}$ & & Universal exhaust-gas system \\
$\mathrm{V}$ & {$\left[\mathrm{m}^{3}\right]$} & Chamber volume \\
VGT & & Variable geometry turbine \\
$\mathrm{w}$ & {$[\mathrm{J} /(\mathrm{deg} * \mathrm{~K})]$} & Weighting factor for $\mathrm{T}_{\text {mean }}$ to take into account wall heat losses in HRR determination \\
$\alpha$ & {$[\mathrm{deg}]$} & Crank Angle coordinate \\
$\gamma$ & {$[\mathrm{adm}]$} & Ratio between constant pressure and constant volume specific heat \\
$\eta_{\mathrm{v}}$ & {$[\mathrm{adm}]$} & Volumetric efficiency \\
$\lambda_{\mathrm{UEGO}}$ & {$[\mathrm{adm}]$} & Dimensionless air to fuel ratio measured by UEGO sensor \\
& &
\end{tabular}

\section{Experimental setup}

\subsection{Hardware plant and control system}

Every experimental data presented from now on has been acquired on a 1.3 liters common rail diesel engine mounted in a test cell whose parameters are reported in Table 1.

\begin{tabular}{|c|c|}
\hline Displacement & 1.248 liters \\
\hline Architecture & $\mathrm{L} 4$, firing order $1-3-4-2$ \\
\hline Maximum Power & 70 kW@3800rpm \\
\hline Maximum Torque & 200 Nm@1500 rpm \\
\hline Compression Ratio & $16.8: 1$ \\
\hline Number of valves & 4 per cylinder \\
\hline Injection system & Common Rail, Multijet \\
\hline Intake system & Turbocharged with variable geometry turbine \\
\hline Exhaust Gas Recirculation & High pressure, liquid cooled \\
\hline
\end{tabular}


Four piezoelectric in-cylinder pressure transducers (AVLGH14P) have been fitted in the glow plugs hole through proper adapters Each signal, conditioned by a charge amplifier (mASTRO by Alma Automotive), is acquired and processed by an indicating system (OBI by Alma Automotive). The engine is equipped with a development ECU that allows access to main control parameters, actuators setpoints and sensors readings through ETAS INCA software. In addition, an ASAM/ASAP communication has been set up between test bed control system and INCA itself in order to implement some of the test conduction and management strategies reported in the next section. Fuel consumption is monitored using an AVL 733s balance and exhaust NOx concentration is measured with a Continental NOx sensor for on-board application. A laboratory intake air mass flow rate sensor (FEV DN/100) has been installed in some tests to determine MAF accuracy and precision in several operating conditions. The standard exhaust aftertreatment system has been completely removed. The scheme of the complete engine installation is reported in Fig. 1, where every sensor (italic) and data managing system (bold) used for the following analysis is shown.

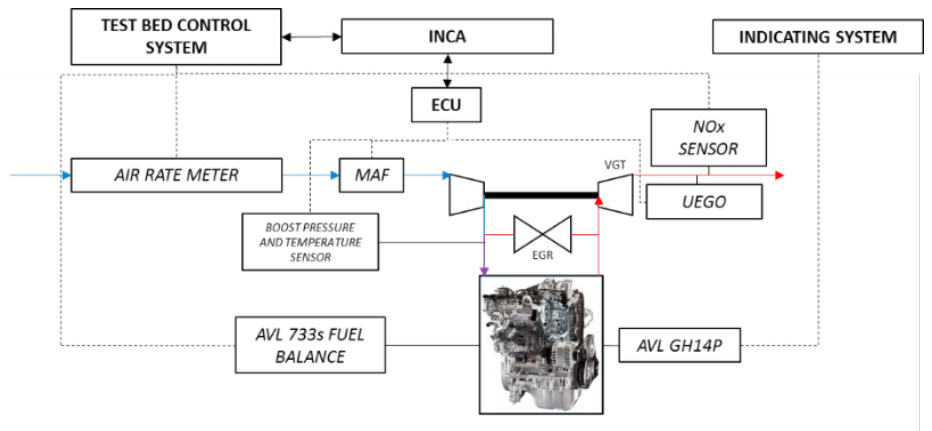

Fig. 1: Experimental Setup

\subsection{Test plan}

The experimental campaign has been primarily focused on producing data suitable to characterize the model response to the control parameters that have a significant effect on exhaust NOx concentration, both on the fuel and and air side, which means that for each operating point, namely engine speed (rpm) and load (BMEP), a $3^{3}$ steadystate DOE has been performed taking into account fresh air setpoint, boost pressure and injection timing variation. For each combination of the other parameters, the highest level of air setpoint has always been set to the maximum allowable, to ensure a null EGR rate. This operation has been necessary to obtain a volumetric efficiency look-up table, which is a fundamental model calibration data as it will be clear in the next section. Furthermore, to make the complete and independent control of air and boost pressure, a PI controller has been implemented in the test bed control system environment, which outputs a VGT position correction to follow a predetermined boost pressure target. With regard to the management of the air mass flow, it is assigned to the standard ECU software: the targets have been overwritten and the EGR valve opening has been consequently adjusted. Regarding the injection pattern, the nominal calibration is applied, no variations have been imposed to the number of injection, dwell time or fuel quantity split: a three-injection pattern is adopted in general, two injections are used at high loads and speed. In Table 2, factors ranges are listed in detail.

\begin{tabular}{ll} 
Table 2: Test plan ranges \\
\hline Engine Speed [rpm] & $1800 \div 3000$ \\
Engine Load, BMEP [bar] & $5 \div 13$ \\
Injection Timing [ $\Delta$ CA with respect to baseline] & $-4 \div 4$ \\
Boost Pressure [ $\Delta \%$ with respect to baseline] & $-10 \div 10$ \\
Mass Air Flow [ $\Delta \%$ with respect to baseline] & $-10 \div 0, \max$ \\
\hline
\end{tabular}


Moreover, specific tests have been performed to validate the model in transient condition: load step, engine speed ramp, start of injection step and EGR ramp are shown in the results section.

\section{Model structure}

As previously stated, the base model expression representing the first step of the present discussion is the one developed by Arrègle et al. [5] and recalled in equation (1):

$$
N O x_{m}=\int_{S O C}^{E O C} k_{1} \cdot H R R(\alpha) \cdot\left(\frac{n}{2000}\right)^{k_{2}} \cdot e^{\left(\frac{k_{3}}{T_{a d}(\alpha)}\right)} d \alpha
$$

where: $N O x_{m}$ is the exhaust nitrogen oxides mass, $\alpha$ is the crank angular coordinate, SOC and EOC are the angle at which the combustion starts and ends respectively, $n$ is the engine speed, $H R R$ and $T_{a d}$ are the heat release rate and adiabatic flame temperature resolved on angle basis, $k_{1}, k_{2}, k_{3}$ are constant tuning parameters. The idea behind the above expression is to describe thermal NOx reaction kinetics with an Arrhenius-like function instantaneously weighted with the amount of energy delivered by combustion, therefore some minor contribution to NOx origination, such as prompt NOx, are neglected. Likewise, Equation 1 does not provide any sensitivity to the reentrant NOx (through EGR) reduction process which is not considered in this work.

In this formulation, $H R R$ is intended as the gross heat release rate, thus both heat exchanged to the cylinder walls and blow-by mass have to be included, in literature several empirical correlation model are present to determine this parameters (Annands', Woschni's correlations, for instance). In the present treatment, for calibration simplicity, wall heat losses are assumed proportional to the mean chamber temperature and the total heat produced by combustion is supposed equal to the diesel lower heating value (LHV) multiplied by the injected fuel mass. $H R R$ is then computed as described in equations $(2-5)$.

$$
\begin{aligned}
& \operatorname{HRR}(\alpha)=N H R R(\alpha)+w \cdot T_{\text {mean }}(\alpha) \\
& \operatorname{NHRR}(\alpha)=\frac{\gamma}{\gamma-1} p(\alpha) \frac{d V(\alpha)}{d \alpha}+\frac{1}{\gamma-1} V(\alpha) \frac{d p(\alpha)}{d \alpha} \\
& w=\frac{\left(L H V \cdot m_{\text {diesel }}-\max (C H R)\right)}{\int_{\text {SOC }}^{E O C} T_{\text {mean }}(\alpha) d \alpha} \\
& \operatorname{CHR}(\alpha)=\int_{\text {SOC }}^{\text {EOC }} \operatorname{NHRR}(\alpha) d \alpha
\end{aligned}
$$

Where: $T_{\text {mean }}$ is the mean chamber temperature, $p$ and $V$ are the chamber pressure and volume, $\gamma$ is the ratio between gas specific heat at constant pressure and at constant volume, $m_{\text {diesel }}$ is the injected fuel mass. As it can be noticed, a common representation of the net heat release rate (NHRR) is used as a base contribution, then the shape of the mean temperature, scaled by a factor which allows matching the HRR integral with the amount of energy introduced into the system, is added.

Concerning adiabatic flame temperature, the methodology proposed in [5] has been fully replicated. It is calculated as the sum of three terms: 1) unburnt gas temperature, 2) temperature increment due to combustion, 3) temperature drop caused by dissociation effects. Detailed adiabatic flame temperature estimation procedure can be found in the reference. It is thus fundamental to highlight that a key role is played by the oxygen fraction at the engine intake since it has a great impact on the temperature shift due to combustion. Its value can be determined once the fresh air mass is known (by MAF) and EGR mass is computed [9,10]. The simplest way to estimate the latter component is by subtracting fresh air mass to the global cylinder trapped one. For each operating condition, the cylinder trapped 
mass is obtained by interpolating a look-up table providing the desired value in function of engine speed and boost pressure, calibrated with MAF by forcing a null EGR rate.

The model training dataset has been chosen as a half of the previously depicted experimental plan to perform a global $k_{1}, k_{2}, k_{3}$ optimization, where RMSE has been used as minimization function; results, in terms of part per million mass, are reported in Fig. 2a where training and validation datasets are put in evidence. Even though the distribution is centered on the measured value, a mean error value of $3.5 \mathrm{ppm}$ is present, a clear dispersion is observed while increasing the engine-out NOx concentration. Nevertheless, if data points with a different start of injection (SOI) shift (with respect to the nominal calibration) are grouped, three different zones are identified showing that the combustion phase effect is not detected properly by the model. Moreover, the impact deriving from the second factor in Equation (1), namely the influence of engine speed, is found to be minimal.


Fig. 2: (a) Starting model steady state response, (b) Optimal k3 value vs CA50

These initial considerations led to modifications of the model structure shown in Equation 1: first, engine speed dependency has been removed, secondly a deeper analysis on the " $\mathrm{k} 3$ " parameter has been done which is summarized in the following steps.

- It has been substantiated that if " $\mathrm{k} 3$ " is properly chosen on a single test basis, a value that bring the estimation to match the measured value always exists.

- "k3" has been then computed for each acquired steady-state operating point and a dependency that links optimal " $\mathrm{k} 3$ " value to typical combustion evolving indices has been identified. A clear trend has been found in optimal "k3" vs CA50 distribution Fig. 2b. This correlation suggests that the reaction kinetics has to take into account the angular position at which combustion occurs, CA50 is then the most suitable index to carry the task out.

- This functionality has been "black box" modeled with a linear data fitting.

As a result, Equation 1 has been accordingly revised and turned in the following formulation (Eq. 6):

$$
\begin{aligned}
& \operatorname{NOx}_{m}=\int_{S O C}^{E O C} k_{1} \cdot \operatorname{HRR}(\alpha) \cdot e^{\left(\frac{k_{3}(C A 50)}{T_{a d}(\alpha)}\right)} d \alpha \\
& k_{3}(C A 50)=b_{1} \cdot C A 50+b_{2}
\end{aligned}
$$

\section{Results}

Fig. 3 shows the new model response to the same input used for Fig. 2 a where $b_{1}$ and $b_{2}$ have been globally optimized over the training dataset (Fig. 2b) and $k_{1}$ is maintained the same as the previous result. NOx sensor 
accuracy range is also displayed. As it can be noticed, the data groups representing the three SOI shifts with respect to the nominal engine calibration tend to converge.

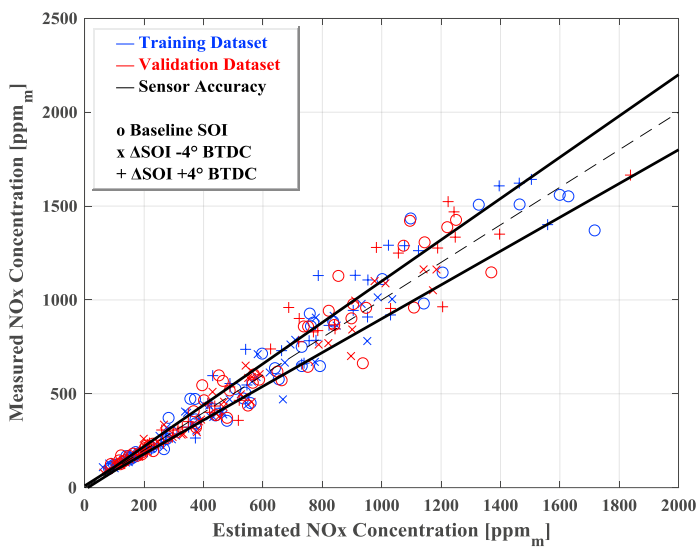

Fig. 3: Revised model steady state response

Although root mean square error (RMSE) improves $\left(166 \mathrm{ppm}_{\mathrm{m}}\right.$ to $99 \mathrm{ppm}_{\mathrm{m}}$ ) while moving from the model shown in Equation 1 to the modified one Eq. (6-7), a certain estimation dispersion is observed. This phenomenon suggests a model sensitivity analysis with respect to input values shift, namely engine-mounted sensors precision. In Fig. 4, two data points are chosen at high and low exhaust NOx concentration. Variations are forced to the principal inputs values in order to quantify the NOx estimation error only due to measure uncertainties. In particular, a $+/-2 \%$ offset is imposed to volumetric efficiency, MAF reading, diesel injected mass, boost temperature and pressure, UEGO measure respectively, by changing one factor at time. Results show a huge impact of every variable that concerns air mass (thus available oxygen) on NOx estimation as one can expect, while fuel mass affected data do not influence model output in a significant way since the amount of energy delivered is detected by the in-chamber pressure sensor.
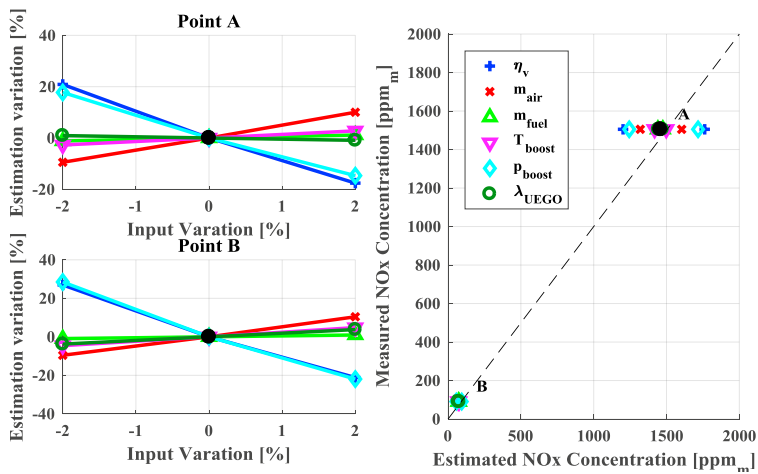

Fig. 4: Model response to input uncertainties

To ensure a complete verification of the adopted formulation a set of transient test have been performed. In this section results are reported where the engine undergoes two operating points variation in terms of load and speed and two calibration parameters changes, $\mathrm{SOI}$ and $\mathrm{m}_{\text {air. }}$. The last couple of variables has been chosen because are considered representative, since they are a quite considerable contribution, in different ways, to the amount of NOx produced. In particular, the former influences the peak local temperatures reached during combustion, while the latter is responsible of the oxygen mass available. In Fig. 5 it can be noticed that the model is able to describe reasonably well the trend followed by the measured value. Some discrepancies (bias) are observed, whose origin could be sought in sensors precision, as shown before. In addition, since it is resolved on a single cycle basis, the estimation dynamic is faster than the sensor one (NOx sensor time constant $\sim 2 \mathrm{~s}$ ) and the transport delay effect is 
avoided. Cyclic dispersion is present instead.
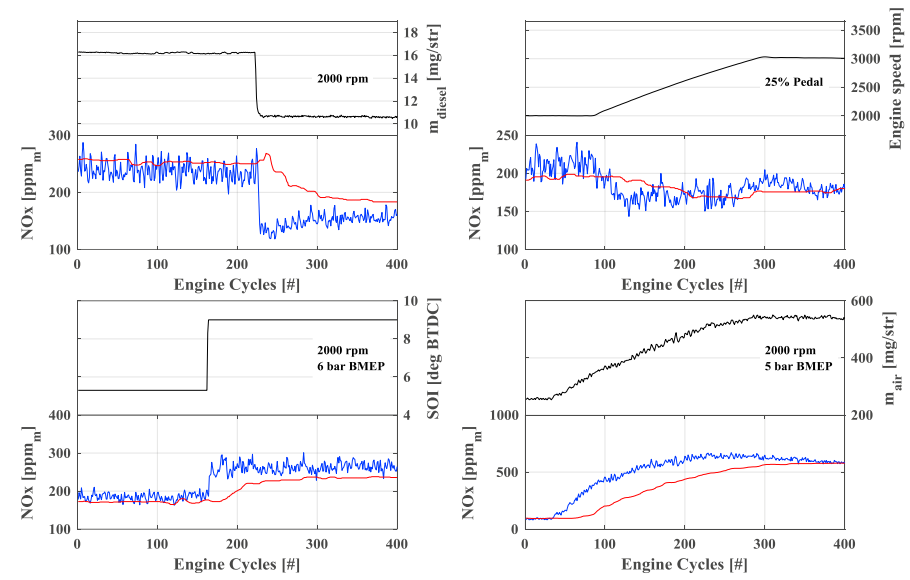

Fig. 5: Proposed model response while performing four kind of transients, red line: measured NOx concentration, blue line: estimated NOx concentration

\section{Conclusions and future work}

The target of this research was to develop some improvement to a model that has been found serviceable to predict NOx exhaust mass and verify its validity when several kind of transients are performed. Fig. 3 shows that the modified model structure is effective to provide a consistent output in terms of estimated thermal NOx concentration since the proposed formulation seems to be able to describe formation mechanism with reasonably few tuning parameters: a global scaling coefficient, and two coefficients to describe "k3" tendency with respect to CA50. In transient operating condition, the model behaves convincingly since it outputs acceptable accuracy results and allows overcoming NOx sensor dynamics to some extent. A main issue has been established concerning air mass measure uncertainties and in cylinder trapped mass estimation (EGR mass flow rate consequently), topics which could require further investigation in order to make the model fully reliable. From a validation point of view future work could be focused on testing the described procedure when the engine is run by unconventional combustions such as LTC (low temperature combustion) or unusual engine-out NOx reducing strategies are actuated, water injection for instance.

\section{References}

[1] Quérel, C., Grondin, O., and Letellier, C., "State of the Art and Analysis of Control Oriented NOx Models," SAE Technical Paper 2012-010723, 2012, https://doi.org/10.4271/2012-01-0723.

[2] Kihas, D. and Uchanski, M., "Engine-Out NOx Models for on-ECU Implementation: A Brief Overview," SAE Technical Paper 2015-011638, 2015, doi:10.4271/2015-01-1638.

[3] Chavannavar, P., "Development and Implementation of a Mapless, Model Based SCR Control System," SAE Int. J. Engines 7(2):2014, doi:10.4271/2014-01-9050.

[4] Hollauf, B., Breitschlamdel, B., Sacher, T., Hlaxlser, H. et al., "Highest NOx Conversion in SCR Catalysts through Model Based Control," SAE Technical Paper 2011-26-0042, 2011, https://doi.org/10.4271/2011-26-0042.

[5] Arrègle, J., López, J., Guardiola, C., and Monin, C., "Sensitivity Study of a NOx Estimation Model for On-Board Applications, " SAE Technical Paper 2008-01-0640, 2008, https://doi.org/10.4271/2008-01-0640.

[6] C. Guardiola, J. Martín, B. Pla, P. Bares, Cycle by cycle NOx model for diesel engine control, Applied Thermal Engineering, Volume 110, 2017, Pages 1011-1020.

[7] C. Guardiola, J.J. López, J. Martín, D. Garcia-Sarmiento, Semiempirical in-cylinder pressure based model for NOX prediction oriented to control applications, Applied Thermal Engineering, Volume 31, Issue 16, 2011, Pages 3275-3286.

[8] Carlucci, A., Benegiamo, M., Camporeale, S., and Ingrosso, D., "Improvement of the Control-Oriented Model for the Engine-Out NOX Estimation Based on In-Cylinder Pressure Measurement," SAE Technical Paper 2017-24-0130, 2017, doi:10.4271/2017-24-0130.

[9] Yang, Z., Winward, E., O'Brien, G., Stobart, R. et al., "Modelling the Exhaust Gas Recirculation Mass Flow Rate in Modern Diesel Engines," SAE Technical Paper 2016-01-0550, 2016, doi:10.4271/2016-01-0550.

[10] Guardiola, C., Pla, B., Blanco-Rodriguez, D., and Bares, P., "Cycle by Cycle Trapped Mass Estimation for Diagnosis and Control," SAE Int. J. Engines 7(3):2014, doi:10.4271/2014-01-1702. 